EPJ Web of Conferences 59, 17018 (2013)

DOI: $10.1051 /$ epjconf/20135917018

(C) Owned by the authors, published by EDP Sciences, 2013

\title{
Electron energy distributions through superdense matter by Monte-Carlo simulations
}

\author{
A. Okabayashi ${ }^{1,2, a}$, H. Habara ${ }^{1,2}$, T. Yabuuchi ${ }^{1,2}$ and K.A. Tanaka ${ }^{1,2}$ \\ ${ }^{1}$ Graduate School of Engineering, Osaka University, 2-1, Yamada-oka, Suita, Osaka \\ 565-0871, Japan \\ 2 Institute of Laser Engineering, Osaka University, 2-6, Yamada-oka, Suita, Osaka 565-0871, \\ Japan
}

\begin{abstract}
We have studied energy distribution of fast electrons passing through a highly compressed core plasma for fast ignition research in inertial confinement fusion. Recent PIC calculations indicate that the collective effect of electric and magnetic fields on the transport may be less significant than the binary collisions in the case of a high density fusion pellet. In order to understand the net effect of binary collisions in dense plasma, we calculate electron energy distributions at several viewing angles using an electromagnetic cascade Monte-Carlo simulation, EGS5, for estimation of the contribution of multi collisional process. Here, the construction of physical parameters in the code were taken from the calculation results given by 2 dimensional particle-in-cell simulations.

In the result, the number of electrons detected on the laser axis within the range to $15 \mathrm{MeV}$ significantly decreases for the superdense region $\left(\max : 1.6 \cdot 10^{25}\left[/ \mathrm{cm}^{3}\right]\right.$ ) compared with the low density plasma. The reduction on the electron number decreases with increase of observation angles gradually and finally the number almost coincides more than 40 degrees.
\end{abstract}

\section{INTRODUCTION}

In Fast ignition [1] in inertial confinement fusion research, it is one of the key issues to heat the compressed core plasma by fast electrons generated via ultraintense laser pulse. Therefore, the estimation of electron energy distributions is crucially important for understanding of heating processes to the core plasma.

The required energy of fast electrons ranges from hundreds of keV to several $\mathrm{MeV}$ at the core. In addition high current density is necessary to heat extremely high density plasma sufficiently for ignition of the fuel. In the classical framework, physical processes when electrons lose its energy via the collision in plasmas are considerably complicated. Recently, the importance of binary collisions at the core plasma has been indicated by PIC simulations [2, 3] compared with the collective effects of electric and magnetic fields on high density fusion pellet. Therefore, it is necessary to take into account binary collisions at dense plasma in order to understand how high density electron current transports in experiments [4-7]. Furthermore, the energy distribution of fast electrons has not been well understood inside plasma. From these points of view, we calculated electron energy distributions at spatially separated degree through the superdense matter by the Electron Gamma Shower 5 (EGS5) [8] program based on the parameters from two dimensional particle-in-cell (2DPIC) code [9]. EGS5 [8] is an electromagnetic cascade Monte-Carlo code for electron and photon transportation calculation in a wide-range of energies in arbitrary geometry. When electrons pass in the material, energy is lost

\footnotetext{
ae-mail: okabayashi-a@eie.eng.osaka-u.ac.jp
}

This is an Open Access article distributed under the terms of the Creative Commons Attribution License 2.0, which permits unrestricted use, distribution, and reproduction in any medium, provided the original work is properly cited. 
via ionization and also electron propagation direction is shifted by multiple scattering, resulting that the electromagnetic cascade shower happens. In EGS5, the type of Fredholm transportation equation is used as a basis for the electromagnetic cascade shower and can treat multiple scattering under completely ionized plasma.

\section{SIMULATION SET UP}

EGS5 requires input parameters such as incident particles, material compositions, geometry, and the physical mechanism taking place in the object substance. In our calculations, the superdense material geometry [Fig. 1(a)] was taken from the high density plasma core at the integrated FI experiment [4] at Institute of Laser Energy, Osaka University. As shown from Fig. 1(a), fast electrons enter in this green region from $50 \mu \mathrm{m}$ away from the core center. The energy distributions are measured at several viewing angles far away from the superdense matter. Multiple scattering, bremsstrahlung, Møller scattering, and electron pair creation, Compton scattering, Rayleigh scattering, and photoelectric absorption were considered as physical mechanisms under completely ionized plasma.

\subsection{Initial particle condition}

Regarding the incident particle condition, energy distribution and angular dependency of fast electrons inside plasma are applied as calculated by 2D PIC [Fig. 1(b,c)]. In these simulations, the laser wavelength, initial laser intensity and peak laser intensity are set from the experiments. But a smaller spot size $2.5 \mu \mathrm{m} \phi$ and shorter pulse duration (500 fs) are used, and the effect of ionized gold cone is excluded due to our computational restrictions.

In actual condition the laser enters into the material with a wide range of incidence angles depending on laser intensity [10]. Therefore, the dependence of incident angle on the electron number was also given by 2D PIC [Fig. 1(d)]. In this figure, y-position indicates spot size at Monte-Carlo simulation.

\subsection{Material condition}

The density profile of superdense matter is given by Gaussian fitting to the experimental data point [11]. In Fig. 1(e), position 0 indicates core center.

A certain suitable interval was applied because it is difficult to define the density and the material composition by a continuous function in programming material composition data in EGS5 simulations. In space, the core sphere (within $\sim 25 \mu \mathrm{m}$ ) is divided into 10 sections in each direction. In addition, nine electron density districts are defined by equal density intervals in the core. In these regions, the average of mass density was $50 \mathrm{~g} / \mathrm{cm}^{3}$. The core plasma is surrounded by lower density plasma $\left(10^{21}-10^{22}\right)$. As the comparison, the "homogeneous" plasma is defined as a density of $10^{21}\left[/ \mathrm{cm}^{3}\right]$ in whole region. The substance was made of $\mathrm{C}_{8} \mathrm{D}_{8}$ to make $\mathrm{CD}$ shell construct matter.

\section{RESULTS AND DISCUSSIONS}

\subsection{Inhomogeneous vs homogenous}

The electron energy distributions are shown in Fig. 1(f-h). At 40 degrees, the electron spectra are nearly identical in spite of presence of super-dense region. The reason is that the electrons pass only low density area less than $10^{23}\left[/ \mathrm{cm}^{3}\right]$. On the other hand, at 20 degrees, the electrons gradually begin to pass high density area from $10^{24}$ to $10^{25}\left[/ \mathrm{cm}^{3}\right]$. The electrons within $5 \mathrm{MeV}$ stop in the plasma due to multiple scattering by the collision. On the other hand, the high energy electron can pass through. The behavior of low-energy electrons taken from the track data is shown in Fig. 1(i). At 0 degree observation angle, wide energy range of electrons up to $15 \mathrm{MeV}$ suffer the scattering as passing high density area about 
(a)

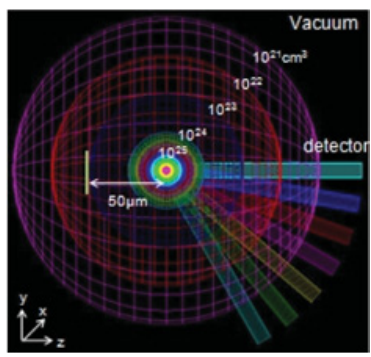

(b)

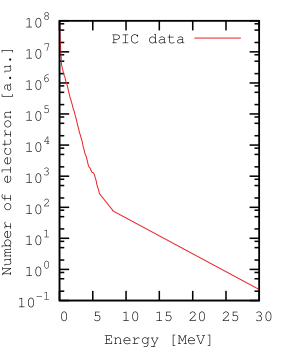

(e)

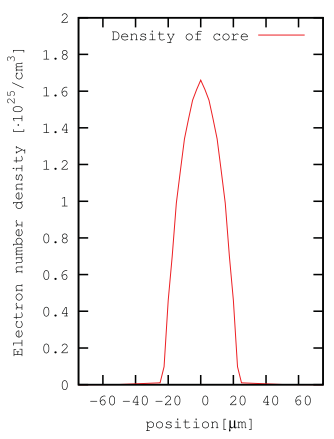

(i)

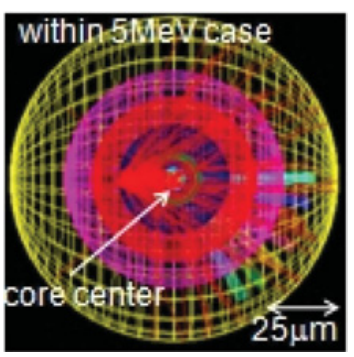

(f)

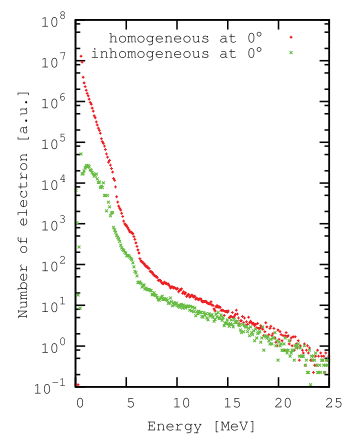

(j)

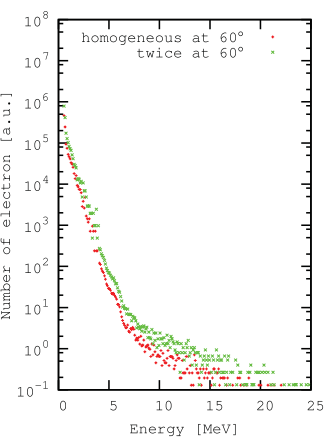

(c)

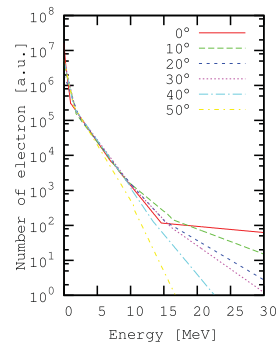

(g)

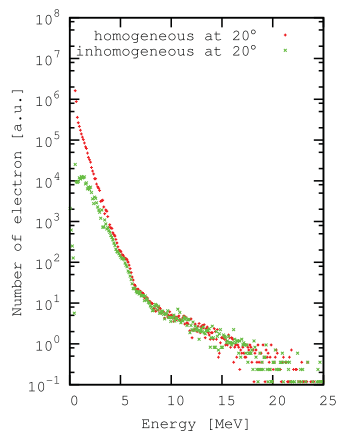

(k)

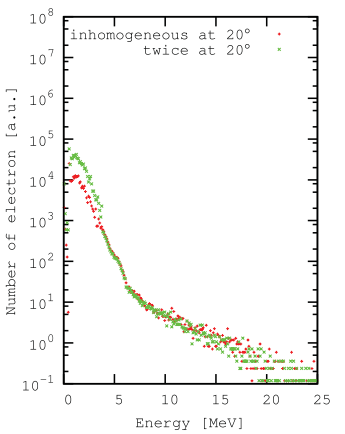

(d)

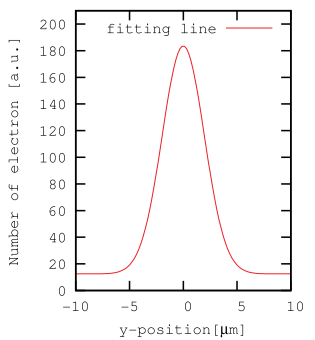

(h)

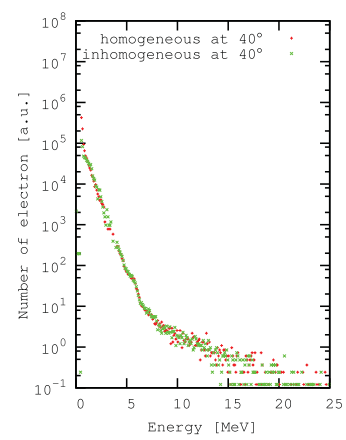

(1)

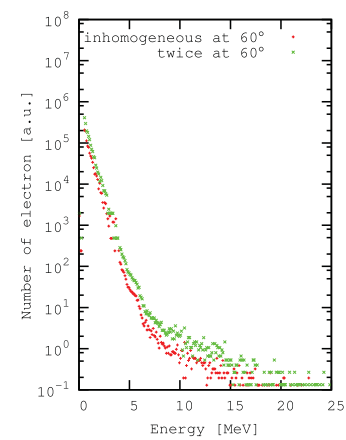

Figure 1. (a) Calculation geometry in EGS5. (b) Initial electron spectrum by 2 DPIC, (c) for different angles. (d) Spatial distribution of electrons. (e) Density profile of core plasma. Calculated electron energy spectra for experimental condition: (f) at $0^{\circ}$, (g) at $20^{\circ}$, (h) at $40^{\circ}$. (i) Electron track data within $5 \mathrm{MeV}$ case. In the emission divergence to be twice, electron spectra: (j) for homogeneous plasma at $60^{\circ}$, for superdense core (k) at $20^{\circ}$, (1) at $60^{\circ}$.

$10^{25}\left[/ \mathrm{cm}^{3}\right]$. For $20^{\circ}$ spectra [Fig. $\left.1(\mathrm{~g})\right]$, the number of electrons from several to $5 \mathrm{MeV}$ is nearly halved compared with the homogeneous case, and this is in good agreement with experimental result [5]. Note that the number of electrons less than 2 or $\mathrm{MeV}$ completely disagrees with the experiment due to lack of electrostatic field in the calculation, to pull back the electrons into the plasma especially for lower energy electrons. On the other hand, the number over $5 \mathrm{MeV}$ does not show any significant reduction in the simulations whereas the experiment shows a reduction.

From these results, the modification on electron energy distribution strongly depends on the plasma density and the mean free path. Of course there is a possibility that the actual density in the experiment 


\section{EPJ Web of Conferences}

is higher than assumed in the simulation to explain this disagreement. As another reason, anomalous stopping as a collective process can happen against steep density gradient $[6,12,13]$, resulting in turbulent magnetic energy damping.

Thus, it is interesting to consider the relation between the energy distribution and the energy deposition of electrons. Therefore, we will calculate the distribution of electron energy deposition in the core plasma, and discuss the relation to the electron energy distributions through the core in the near future.

\subsection{Effect of emission divergence}

It is also interesting to consider the difference of divergence angle on initial spectrum without changing other conditions. The emission divergence up to 30 degrees was artificially enhanced twice while keeping the total energy of electrons. The calculation results are shown in Fig. 1(j-1). In low, homogeneous density case [Fig. 1(j)], the electron number detected at larger than 40 degrees has increased due to artificial enhancement of electrons for the angles. On the other hand, in an inhomogeneous case, because the electron energy distribution within $20^{\circ}$ observation angles does not change so much, low energy electrons up to several $\mathrm{MeV}$ for 20 degrees can pass lower density plasma compared with the normal case, resulting in the enhancement on electron number. For $60^{\circ}$ electrons, the number increases in high energy range more than $10 \mathrm{MeV}$ as the same reason with Fig. 1(j).

\section{CONCLUSION}

First, we estimated the modification of electron energy distributions at different viewing angles. In the calculation, we show that the number of electrons depends on the plasma density they pass through. This dependency can be seen from particle track data and is good agreement with experimental data [5, 6] within several $\mathrm{MeV}$ electrons even the binary collisional process is considered. However, at 20 degrees case, electron spectra over $5 \mathrm{MeV}$ are discordant with the experiment. Here, it is necessary to consider the steep energy damping mechanism such as anomalous stopping.

Secondary, we estimated effects of the divergence angles on the spectra. In a uniform density profile, energy distributions directly depend on an artificial enhancement in the initial angular spectra. On the other hand, in an inhomogeneous case, we can also see that the electron number depends on the plasma density and initial angular distribution given by 2D PIC.

In the near future, we will calculate the energy deposition on core plasma by using a full scale PIC simulation to understand anomalous stopping process including the steep energy dissipation mechanism.

The authors greatly thank H. Sakagami at National Institute for Fusion Science. Moreover, we thank T. Iwawaki at University of Osaka for helpful discussions. And, the author is supported by JSPS Research Fellowship for young scientists. A part of this study is supported by core-to-core program of International Collaboration for High Energy Density Science (ICHEDS) and Asian core program for high energy density science using intense laser photons (ASHULA) supported of JSPS.

\section{References}

[1] M. Tabak, J. Hammer, et al., Phys. Plasma, 1, 1626 (1994)

[2] Y. Sentoku, K. Mima, et al., Phys. Rev. Letts, 90, 155001 (2003)

[3] A. J. Kemp, Y. Sentoku, et al., Phys. Rev. Letts, 97, 235001 (2006)

[4] R. Kodama, H. Shiraga, et al., Nature, 418, 933 (2002) 


\section{IFSA 2011}

[5] T. Yabuuchi, K. Adumi, et al., Physics of Plasmas, 14, 040706 (2007)

[6] T. Yabuuchi, A. Das, et al., New J. Phys, 11, 093031 (2009)

[7] T. Iwawaki, H. Habara, et al., Review of Scientific Instruments, 81, 10E535 (2010)

[8] KEK, SLAC, and the University of Michigan teams, SLAC Manual SLAC-R-730, (2007)

[9] H. Sakagami and K. Mima, Proc. of 2nd Int. Conf. Inertial Fusion Sciences and Applications, 380-383 (Elsevier, 2002)

[10] Y. T. Li, J. Zhang, et al., Phy. Rev. E, 69, 036405 (2004)

[11] R. B. Campbell, R. Kodama, et al., Phy. Rev. Lett, 94, 055001 (2005)

[12] S. K. Yadav, A. Das, et al., Phys. Plasmas, 15, 062308 (2008)

[13] S. K. Yadav, A. Das, et al., Phys. Plasmas, 16, 040701 (2009) 